\title{
Study on knowledge, attitude and practice of ante-natal care among pregnant women attending antenatal tertiary care institution
}

\author{
Vuppu Sitalakshmi*, Pothu Bavyasri, Rajyalakshmi Talapala, Manjula Kopperla
}

Department of Obstetrics and Gynecology, Narayana Medical College and Hospital, Nellore, Andhra Pradesh, India

Received: 02 January 2020

Accepted: 07 February 2020

\section{*Correspondence:}

Dr. Vuppu Sitalakshmi,

E-mail: research.nmch@rediffmail.com

Copyright: (C) the author(s), publisher and licensee Medip Academy. This is an open-access article distributed under the terms of the Creative Commons Attribution Non-Commercial License, which permits unrestricted non-commercial use, distribution, and reproduction in any medium, provided the original work is properly cited.

\begin{abstract}
Background: The primary aim of antenatal care is to achieve a healthy mother and baby. Antenatal care helps to identify the complications and to remove anxiety and dread associated with delivery. Current study designed to assess knowledge, attitude and ante natal care practice among pregnant women attending to antenatal tertiary care hospital.

Methods: A cross-sectional study was conducted on 500 pregnant women attending antenatal care centre at department of obstetrics and genecology, Narayana Medical College and Hospital, Nellore during June 2017 to June 2019 under Narayana Mathru Seva Pathakam. Face to face interview was conducted to assess their knowledge, attitude and practice using a structured questionnaire.

Results: A total $86 \%$ belongs to $20-29$ years age group and $71.4 \%$ belongs to multiverdia. $20.6 \%$ women responded that they had completed 3 antenatal visits, $26.6 \%$ are those who thought that at least more than 5 ANC checkup should be done during pregnancy. $96.1 \%$ pregnant responded that registration for antennal care and visits is essential. $87.2 \%$ responds to take iron and folate during pregnancy and $11 \%$ responds does not taken the same. $92 \%$ pregnant responded that tetanus toxoid (TT) should be given during pregnancy but $3.2 \%$ told don't know. When they asked about resting period, $24.2 \%$ responded 5-6 hours, 35.4\% responded 7-8 hours, 35.6\% responded > 8 hours per day to rest they are taking. $55.8 \%$ pregnant responded that they had taken decision to give birth the baby in the hospital. $71.2 \%$ pregnant females reported that they knew about the danger signs during pregnancy. The reasons for not attending antenatal check-up were financial reasons, illiterate (15.4\%), transport and unaware about ANC.

Conclusions: Information, education and communication activities should be increased on ANC through community campaign and mass media to motivate women to utilize maternal care services.
\end{abstract}

Keywords: Antenatal care, Attitude, Danger signs, Knowledge, Practices, Pregnant women

\section{INTRODUCTION}

Appropriate antenatal care (ANC) is one of the pillars of maternal and perinatal health. Antenatal care is considered as a backbone of obstetrical services and health of pregnant women. It includes not only providing nutrition and care but also detection and management of maternal and fetal complications during ANC period ANC is the care a woman receives throughout her pregnancy in order to ensure that both the mother and child remain healthy. Almost $90 \%$ of maternal deaths occur in developing countries and over half a million women die each year due to pregnancy and childbirth related causes. ${ }^{1}$ Annually, 5 lakh women die globally as a result of pregnancy and childbirth. ${ }^{2}$

In India, it is heartening to note that maternity mortality rate has declined from 212 in 2007-2009 to 178 in 201012. ${ }^{2}$ The World Health Organization estimates that 300 million women in the developing world suffer from shortterm or long-term morbidities brought about by pregnancy and childbirth. The current maternal mortality 
ratio in India is 167/100,000 live births (2011-2013), whereas the country's millennium development goal in this respect is $109 / 100,000$ live births by 2015 . High levels of infant mortality (50/1000 births), neonatal mortality (29/1000 live births), and maternal mortality (167/100,000 live births), and lower levels of deliveries with skilled assistance (45\%-NFHS-3) remain major public-health challenges in India. ${ }^{3,4}$ Understanding maternal knowledge and practices of the community regarding care during pregnancy and delivery are required for program implementation. ${ }^{5}$

Knowledge refers to a pregnant women's understanding of components of antenatal care which include registration of pregnancy, danger signs during pregnancy, intake of prophylactic Iron and Folic Acid tablets during pregnancy and adapting family planning methods. Attitudes are emotional, motivational, perceptive and cognitive beliefs that positively or negatively influence the behaviour or practice of an individual. A pregnant female's antenatal check-up, adapting family planning behaviour is influenced by her emotions, motivations, perceptions and thoughts. Practices are defined as the observable actions of a pregnant women that could affect her to go to the hospital for antenatal check-up, after knowing the danger signs during pregnancy, how she is making the arrangement to attend hospital and how she had adapting the family planning methods after marriage, in the previous and present pregnancy.

Data related to this very important issue are scarce or less in Andhra Pradesh state. In spite of all efforts to reach and register every antenatal mother, the results are not up to desired level. Current study was conducted to determine the level of knowledge, attitude, and practice related to ANC among the pregnant women and to assess the awareness about their own health during pregnancy. This may be useful to further planning of health intervention program.

\section{METHODS}

A cross-sectional study was undertaken to assess the knowledge, attitude, and practices regarding ANC among pregnant women attending to department of obstetrics and genecology, tertiary Care teaching Hospital Narayana Medical College, Nellore, Andhra Pradesh from June 2017 to June 2019 under Narayana Mathru Seva Pathakam.

Demographic characteristics namely age, parity, type of family, education and occupation, and socioeconomic status (SES) were selected for studying association with knowledge, attitude and practices regarding ANC.

\section{Inclusion criteria}

- all pregnant women came to antenatal check-up and follow-up.

\section{Exclusion criteria}

- pregnant women those were not willing to answer any questions relating to ANC knowledge, attitude and practices.

The data were collected by interviewing all the eligible

subjects willing to participate in the study.

Knowledge was assessed about ANC visits, tetanus immunization, investigations, and nutritional factors, danger signs of pregnancy.

Attitude variables were an opinion on the ANC registration, visits, diseases, dietary changes, and iron and folic acid (IFA) intake and its regularity in the intake.

Practices questions with regards to ANC visit, dietary changes made during pregnancy, folic acid tablets recorded.

\section{Score of knowledge (percentage)}

Percentages used as indicators of knowledge are determined from the numerical indicators.

Score - For a score-based indicator of knowledge, each respondent is given a score based on the number of correct responses provided.

Knowledge score of pregnant will be calculated for each question as the total number of correct responses to one question divided by the total number of responses.

Score of knowledge $=\frac{\text { Sum of correct response given by all respondents }}{\text { Total number of respondents. }}$

Total 13 questions were asked about knowledge aspect of antenatal care, perceived danger signs during pregnancy. Then score for knowledge about antenatal care is calculated.

Attitude score: this will be measured by asking the respondents to judge whether they are positively or negatively inclined towards Antenatal check-up, diseases during pregnancy and diet.

The practices followed by the pregnant female regarding antenatal care, delivery site, occupation and education of pregnant were asked. The questions regarding practices contain 7 questions. Then score for practices regarding antenatal care was calculated.

\section{Statistical analysis}

The data thus collected were tabulated and analysis was done by using SPSS-version 24 version. Descriptive statistics like mean and percentage were used. 
RESULTS

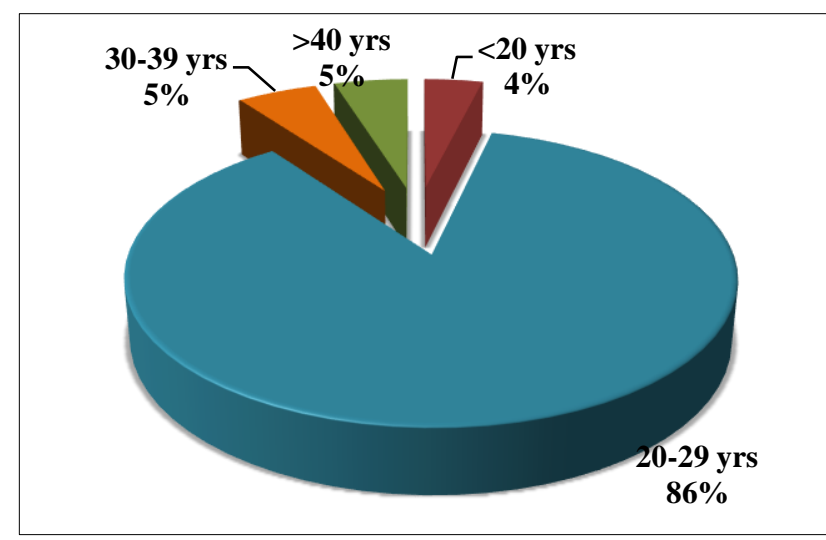

Figure 1: Age wise distribution of pregnant women.

Total number of 500 participants were contacted and interviewed. The mean age of the pregnant were $25 \pm 3.61$ years (range: 18-40 years). Maximum number of pregnant women ( $86 \%$ ) lying between 20 to 29 years age group followed by 30 to 39 years age group (5\%), > 40 years age group $(5 \%)$, and $<20$ years age group $(4 \%)$ respectively (Figure 1).

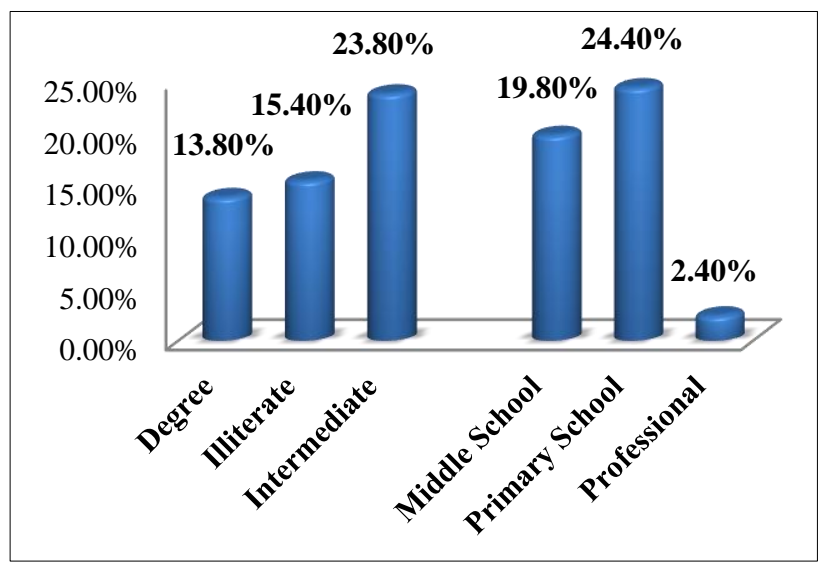

Figure 2: Level of education of the pregnant women.

Table 1: Attitude of pregnant female about antenatal care.

\begin{tabular}{|c|c|c|c|c|}
\hline Q. No. & Questions asked & Option & Frequency & Percentage \\
\hline \multirow{5}{*}{1} & \multirow{5}{*}{$\begin{array}{l}\text { Necessary to register during } \\
\text { pregnancy for antenatal check-up }\end{array}$} & Strongly agree & 171 & 34.2 \\
\hline & & Agree & 232 & 46.4 \\
\hline & & Neutral & 76 & 15.2 \\
\hline & & Dis agree & 14 & 2.8 \\
\hline & & Strongly dis agree & 2 & 0.4 \\
\hline \multirow{5}{*}{2} & \multirow{5}{*}{$\begin{array}{l}\text { Antinatal follow-up is good to monitor mother and } \\
\text { fetus health }\end{array}$} & Strongly agree & 155 & 31.0 \\
\hline & & Agree & 238 & 47.6 \\
\hline & & Neutral & 78 & 15.6 \\
\hline & & Dis agree & 19 & 3.8 \\
\hline & & Strongly dis agree & 4 & 0.8 \\
\hline \multirow{6}{*}{3} & \multirow{6}{*}{ Number of antenatal visits required } & 1 & 30 & 6.0 \\
\hline & & 2 & 100 & 20.0 \\
\hline & & 3 & 103 & 20.6 \\
\hline & & 4 & 81 & 16.2 \\
\hline & & 5 & 49 & 9.8 \\
\hline & & More & 133 & 26.6 \\
\hline \multirow{3}{*}{4} & \multirow{3}{*}{$\begin{array}{l}\text { Number of TT doses received during the present } \\
\text { pregnancy }\end{array}$} & 1 & 54 & 10.8 \\
\hline & & 2 & 386 & 77.2 \\
\hline & & 3 & 43 & 8.6 \\
\hline 5 & What changes in the diet during the present pregnancy & & & \\
\hline \multirow{2}{*}{6} & \multirow{2}{*}{ Are your taking iron and folic acid tablets } & Yes & 436 & 87.2 \\
\hline & & No & 55 & 11.0 \\
\hline \multirow{5}{*}{7} & \multirow{5}{*}{ If yes, number of iron and folic acid tablets } & $<50$ & 53 & 10.6 \\
\hline & & $50-99$ & 133 & 26.6 \\
\hline & & $100-149$ & 202 & 40.4 \\
\hline & & $150-200$ & 96 & 19.2 \\
\hline & & $>200$ & 5 & 1.0 \\
\hline \multirow{4}{*}{8} & \multirow{4}{*}{ How many hours of rest do you take per day } & $5-6$ & 121 & 24.2 \\
\hline & & $7-8$ & 177 & 35.4 \\
\hline & & $>8$ hours & 178 & 35.6 \\
\hline & & Others & 19 & 3.8 \\
\hline
\end{tabular}




\begin{tabular}{|lllll|}
\hline Q. No. & Questions asked & Option & Frequency & Percentage \\
\hline \multirow{2}{*}{9} & \multirow{2}{*}{ Did you ever take medicines without consulting doctor } & No & 244 & 48.8 \\
\cline { 3 - 5 } & & Yes & 246 & 49.2 \\
\hline \multirow{2}{*}{10} & $\begin{array}{l}\text { In current pregnancy, hyper tension, diabetes, thyroid, } \\
\text { anemia }\end{array}$ & No & 308 & 61.6 \\
\cline { 3 - 5 } & \multirow{2}{*}{ If yes } & Yes & 192 & 38.4 \\
\cline { 3 - 5 } & Self-medication & 143 & 28.6 \\
\cline { 3 - 5 } & Hosp & 10 & 2.0 \\
\hline
\end{tabular}

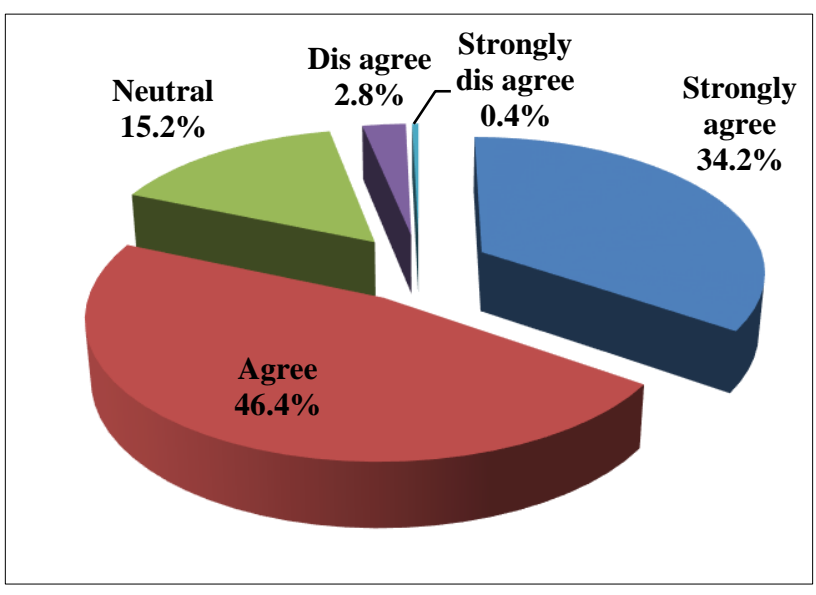

Figure 3: ANC check-up is essential as told by respondent.

Equal number of the respondents lived in nuclear and joint families while a quarter of them lived in rented house. Maximum no. of pregnant women $(71.4 \%)$ possesses their multiverdia and $27.6 \%$ pregnant women are those who possess first pregnancy.

According to Figure 2, maximum no. of respondents (24.4\%) are well educated having Primary school, 23.8\% respondents are those who have intermediate education, $19.8 \%$ respondents are those who have educational qualification till middle School and $13.8 \%$ respondents are those who have qualification till degree, $15.4 \%$ respondents are illiterate, and only $2.4 \%$ respondents are those who have professional qualification (Figure 2). A total 67.2 population belongs to rural and remaining were from urban residence.

Table 1 showing the attitude score of pregnant females about antenatal care. The pregnant females were asked about the benefit they are getting by attending antenatal care centre.

When they asked about regular check-up, 46.2\% respondent agreed, $34.2 \%$ strongly agreed, $15.2 \%$ neutral about that it was necessary to register during pregnancy for antenatal check-up (Figure 3).

According to Figure 3, 100\% respondents are those who are agreed that the ANC check-up is necessary for pregnant women for safe and healthy delivery (Table 1).
According to Table 1, maximum number of respondents (26.6\%) are those who thought that at least more than 5 ANC check-up should be done during pregnancy, and 9.8\% respondents are who thought that at least 5 ANC check-up should be done during pregnancy.

Table 2: Question number 5 regarding the attitude of pregnant female about antenatal care: Changes in the diet during the present pregnancy.

\begin{tabular}{|lll|}
\hline Option & Frequency & Percentage \\
\hline $\mathrm{A}$ & 45 & 9.0 \\
\hline $\mathrm{A}, \mathrm{B}, \mathrm{C}$ & 2 & 0.4 \\
\hline $\mathrm{A}, \mathrm{B}, \mathrm{C}, \mathrm{D}$ & 4 & 0.8 \\
\hline $\mathrm{A}, \mathrm{B}, \mathrm{C}, \mathrm{E}$ & 10 & 2.0 \\
\hline $\mathrm{A}, \mathrm{B}, \mathrm{E}$ & 2 & 0.4 \\
\hline $\mathrm{A}, \mathrm{C}, \mathrm{D}, \mathrm{E}$ & 4 & 0.8 \\
\hline $\mathrm{A}, \mathrm{C}, \mathrm{E}$ & 1 & 0.2 \\
\hline $\mathrm{A}, \mathrm{E}$ & 1 & 0.2 \\
\hline $\mathrm{ALL}$ & 254 & 50.8 \\
\hline $\mathrm{B}$ & 60 & 12.0 \\
\hline $\mathrm{B}, \mathrm{C}$ & 4 & 0.8 \\
\hline $\mathrm{B}, \mathrm{C}, \mathrm{D}$ & 6 & 1.2 \\
\hline $\mathrm{B}, \mathrm{C}, \mathrm{E}$ & 12 & 2.4 \\
\hline $\mathrm{B}, \mathrm{D}$ & 1 & 0.2 \\
\hline $\mathrm{C}$ & 47 & 9.4 \\
\hline $\mathrm{C}, \mathrm{D}$ & 5 & 1.0 \\
\hline $\mathrm{C}, \mathrm{D}, \mathrm{E}$ & 4 & 0.8 \\
\hline $\mathrm{D}$ & 27 & 5.4 \\
\hline $\mathrm{E}$ & 4 & 0.8 \\
\hline Y & 2 & 0.4 \\
\hline $\mathrm{A}$ - intake of diet (Increase/decrease); B - intake of green \\
leaf veg (Increase/decrease); C - intake of milk \\
(Increase/decrease); D - intake of fruits (Increase/decrease); \\
E - intake of meat (Increase/decrease). \\
\hline
\end{tabular}

Maximum number of respondents (87.2\%) are take iron and folic acid tablets during pregnancy and $11 \%$ respondents do not take iron and folic acid tablets during pregnancy. Among these, $10.6 \%$ responded $<50,26.6 \%$ responded 50-99, 40.4\% responded 100-149, 19.2\% responded $150-200$ and $1 \%$ responded $>200$ micrograms of iron and folate tablets were required to maintain their pregnancy according to their condition.

When they asked about resting period, $24.2 \%$ responded 5-6 hours, $35.4 \%$ responded $7-8$ hours, $35.6 \%$ responded $>8$ hours per day to rest they are taking. 
Table 3: Practices of pregnant females on antenatal care.

\begin{tabular}{|c|c|c|c|c|}
\hline Q. No. & Questions asked & Option & Frequency & Percentage \\
\hline \multirow{4}{*}{1} & \multirow{4}{*}{$\begin{array}{l}\text { Gestation period visited health centre for } \\
\text { Antenatal check-up }\end{array}$} & $1-2 \mathrm{M}$ & 84 & 16.8 \\
\hline & & $2-3 \mathrm{M}$ & 183 & 36.6 \\
\hline & & $3-4 \mathrm{M}$ & 180 & 36.0 \\
\hline & & Other & 50 & 10.0 \\
\hline \multirow{2}{*}{2} & \multirow{2}{*}{$\begin{array}{l}\text { Are you regular in your antenatal visits } \\
\text { for check-ups }\end{array}$} & No & 108 & 21.6 \\
\hline & & Yes & 373 & 74.6 \\
\hline & \multirow{4}{*}{ If No, } & Don't feel like & 16 & 3.2 \\
\hline & & Family non cooperation & 51 & 10.2 \\
\hline & & Not in statia & 38 & 7.6 \\
\hline & & Transportation problem & 78 & 15.6 \\
\hline \multirow{4}{*}{3} & \multirow{4}{*}{ Mother's occupation } & Government & 24 & 4.8 \\
\hline & & Home maker & 293 & 58.6 \\
\hline & & Private & 108 & 21.6 \\
\hline & & Working women & 73 & 14.6 \\
\hline \multirow{7}{*}{4} & \multirow{7}{*}{ Husband's education } & Degree & 110 & 22.0 \\
\hline & & High school & 93 & 18.6 \\
\hline & & Illiterate & 31 & 6.2 \\
\hline & & Intermediate & 122 & 24.4 \\
\hline & & Middle school & 72 & 14.4 \\
\hline & & Primary school & 31 & 6.2 \\
\hline & & Professional & 38 & 7.6 \\
\hline \multirow{3}{*}{5} & \multirow{2}{*}{ Occupation of father } & Government & 91 & 18.2 \\
\hline & & Private & 382 & 76.4 \\
\hline & Family income & 8000 Rs. Per month & & \\
\hline \multirow{4}{*}{7} & \multirow{4}{*}{ Place of delivery } & Home & 62 & 12.4 \\
\hline & & Hospital & 279 & 55.8 \\
\hline & & Other & 2 & 0.4 \\
\hline & & PHC & 22 & 4.4 \\
\hline \multirow{8}{*}{8} & \multirow{8}{*}{ If home delivery } & Comfortable conditions at home & 2 & 0.4 \\
\hline & & Easy labour & 5 & 1.0 \\
\hline & & Easy labour & 14 & 2.8 \\
\hline & & Feel shy & 7 & 1.4 \\
\hline & & No co-operation in the family & 4 & 0.8 \\
\hline & & No nearby health facilities & 7 & 1.4 \\
\hline & & Not aware of medical facilities & 7 & 1.4 \\
\hline & & Transportation problem & 18 & 3.6 \\
\hline \multirow{10}{*}{9} & \multirow{10}{*}{ If hospital delivery } & All & 20 & 4.0 \\
\hline & & $\mathrm{B}, \mathrm{D}, \mathrm{F}$ & 1 & 0.2 \\
\hline & & Encouraging family & 7 & 1.4 \\
\hline & & For a healthy child & 151 & 30.2 \\
\hline & & Free care & 29 & 5.8 \\
\hline & & Free care, nearby facility & 1 & 0.2 \\
\hline & & Good hospital services & 22 & 4.4 \\
\hline & & Nearby health facility & 49 & 9.8 \\
\hline & & On health workers motivation & 9 & 1.8 \\
\hline & & Transport problem & 2 & 0.4 \\
\hline
\end{tabular}

When they asked about medication without consulting doctors, $48.8 \%$ responded no and $49.2 \%$ responded yes that they have taken medicines without consulting doctor. When they asked about any ailment, $61.6 \%$ responded no and $38.4 \%$ responded yes that they had hypertension or diabetes or thyroid problem or anemia problems. In these diseases, $28.6 \%$ responded that they are taking selfmedication, $2 \%$ responded that they are consulting to doctors and $8.8 \%$ responded that they ignore these conditions. 
When they asked about the diet, 50.8\% responded that they increased the diet, both veg diet, milk, fruits and meat diet (Table 2).

When the pregnant were asked about the practise related questions, they responded well.

\section{Gestation period visited health centre for antenatal check-up}

When they asked about regular check-ups, Maximum no. of respondents $(74.6 \%)$ are those pregnant women who are regular in ANC check-ups during their pregnancy and remaining respondents are those who are not regular in ANC check-ups during their pregnancy (Table 3). When they asked about what gestation period you visited to health centre for Antenatal check-up, 16.8\% responded 1-
2 months, $36.6 \%$ responded $2-3$ months, $36 \%$ responded 3-4 months of gestation period.

When they asked about occupation, 58.6\% responded as home maker. When they asked about their husband's occupation, $76.4 \%$ responded as private employment. When they asked for husband's education, $22 \%$ responded as degree, $18.6 \%$ responded as high school, $24.4 \%$ responded as intermediate, $7.6 \%$ responded as professional, $14.4 \%$ responded as middle school and $6.2 \%$ responded as illiterate. The average family income 8000 rupees.

When pregnant asked about place of delivery, 55.8\% responded to have hospital based, $12.4 \%$ responded to have home, $4.4 \%$ responded to have primary health centres near to their home.

Table 4: Knowledge among pregnant female about antenatal care.

\begin{tabular}{|c|c|c|c|c|}
\hline Q. No. & Question & Options & Frequency & Percentage \\
\hline \multirow{10}{*}{ Q } & \multirow{10}{*}{$\begin{array}{l}\text { Check-up is necessary when and why? } \\
\text { A - to know the condition of the baby } \\
\text { B - to know the health of the mother } \\
\text { C - to avoid complications } \\
\text { D- for safe delivery }\end{array}$} & A & 82 & 16.4 \\
\hline & & $\mathrm{A}, \mathrm{B}$ & 1 & 0.2 \\
\hline & & $\mathrm{A}, \mathrm{B}, \mathrm{C}$ & 1 & 0.2 \\
\hline & & $\mathrm{A}, \mathrm{B}, \mathrm{D}$ & 1 & 0.2 \\
\hline & & $\mathrm{A}, \mathrm{C}$ & 1 & 0.2 \\
\hline & & All & 10 & 2.0 \\
\hline & & $\mathrm{B}$ & 40 & 8.0 \\
\hline & & $\mathrm{C}$ & 85 & 17.0 \\
\hline & & $\mathrm{D}$ & 135 & 27.0 \\
\hline & & $\mathrm{E}$ & 9 & 1.8 \\
\hline \multirow{4}{*}{1} & \multirow{4}{*}{$\begin{array}{l}1^{\text {st }} \text { antenatal check-up is done during } 1^{\text {st }}-3^{\text {rd }} \\
\text { months }\end{array}$} & Can't say & 57 & 11.4 \\
\hline & & Don't know & 4 & 0.8 \\
\hline & & No & 68 & 13.6 \\
\hline & & Yes & 358 & 71.6 \\
\hline \multirow{4}{*}{2} & \multirow{4}{*}{$\begin{array}{l}\text { Pregnant need to come for antenatal check- } \\
\text { ups throughout the pregnancy }\end{array}$} & Can't say & 49 & 9.8 \\
\hline & & Don't know & 8 & 1.6 \\
\hline & & No & 139 & 27.8 \\
\hline & & Yes & 283 & 56.6 \\
\hline \multirow{4}{*}{3} & \multirow{4}{*}{ Why TT Inj. given to pregnant women? } & $\begin{array}{l}\text { To protect child from } \\
\text { tetanus }\end{array}$ & 160 & 32.0 \\
\hline & & $\begin{array}{l}\text { To protect mother from } \\
\text { tetanus }\end{array}$ & 101 & 20.2 \\
\hline & & Both & 176 & 35.2 \\
\hline & & Don't know & 55 & 11.0 \\
\hline \multirow{4}{*}{4} & \multirow{4}{*}{$\begin{array}{l}\text { How many Inj. TT should be given to } \\
\text { pregnant mother? }\end{array}$} & 1 & 67 & 13.4 \\
\hline & & 2 & 325 & 65.0 \\
\hline & & 3 & 68 & 13.6 \\
\hline & & Don't know & 16 & 3.2 \\
\hline \multirow{6}{*}{5} & \multirow{6}{*}{$\begin{array}{l}\text { Any need for pregnant mother to undergo } \\
\text { the following tests during pregnancy - } \\
\text { blood tests- }\end{array}$} & Hepatitis B and thyroid & 1 & 0.2 \\
\hline & & All tests & 11 & 2.2 \\
\hline & & Blood grouping and typing & 3 & 0.6 \\
\hline & & $\mathrm{HCV}$ & 3 & 0.6 \\
\hline & & $\begin{array}{l}\mathrm{HCV}, \text { hemoglobin, blood } \\
\text { grouping and typing }\end{array}$ & 1 & 0.2 \\
\hline & & Hemoglobin & 4 & 0.8 \\
\hline
\end{tabular}




\begin{tabular}{|c|c|c|c|c|}
\hline \multirow[t]{12}{*}{ Q. No. } & \multirow[t]{12}{*}{ Question } & Options & Frequency & Percentage \\
\hline & & $\begin{array}{l}\text { Hemoglobin, blood } \\
\text { grouping and typing, blood } \\
\text { sugar }\end{array}$ & 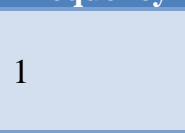 & 0.2 \\
\hline & & $\begin{array}{l}\text { Hemoglobin, blood } \\
\text { grouping and typing, BP } \\
\text { examination }\end{array}$ & 3 & 0.6 \\
\hline & & $\begin{array}{l}\text { Hemoglobin, blood sugar, } \\
\text { BP examination }\end{array}$ & 1 & 0.2 \\
\hline & & Hepatasis B, thyroid & 1 & 0.2 \\
\hline & & Hepatitis B & 13 & 2.6 \\
\hline & & $\begin{array}{l}\text { Hepatitis B, HIV, HCV, } \\
\text { hemoglobin, blood } \\
\text { grouping and typing }\end{array}$ & 1 & 0.2 \\
\hline & & $\begin{array}{l}\text { Hepatitis B, HIV, HCV, } \\
\text { hemoglobin, blood sugar, } \\
\text { BP examination }\end{array}$ & 1 & 0.2 \\
\hline & & HIV & 9 & 1.8 \\
\hline & & Thyroid test: No & 25 & 5.0 \\
\hline & & Thyroid-can't say & 38 & 7.6 \\
\hline & & Thyroid test: Yes & 127 & 25.4 \\
\hline \multirow{3}{*}{6} & \multirow{3}{*}{ Can high PP affect the fetal growth } & Can't say & 26 & 5.2 \\
\hline & & No & 97 & 19.4 \\
\hline & & Yes & 350 & 70.0 \\
\hline \multirow{4}{*}{7} & \multirow{4}{*}{$\begin{array}{l}\text { What are the change to be made in diet of a } \\
\text { pregnant mother }\end{array}$} & Intake of green vegetable & 252 & 50.4 \\
\hline & & Intake of milk & 109 & 21.8 \\
\hline & & Intake of fruits & 114 & 22.8 \\
\hline & & Intake of meat & 16 & 3.2 \\
\hline \multirow{4}{*}{8} & \multirow{4}{*}{$\begin{array}{l}\text { Why iron and folic acid given to pregnant } \\
\text { women? }\end{array}$} & To increase blood of mother & 121 & 24.2 \\
\hline & & To relieve weakness & 86 & 17.2 \\
\hline & & Both & 230 & 46.0 \\
\hline & & Don't know & 51 & 10.2 \\
\hline \multirow{3}{*}{9} & \multirow{3}{*}{$\begin{array}{l}\text { In your opinion where should a pregnant } \\
\text { woman deliver her baby? }\end{array}$} & health care facility/hospital & 311 & 62.2 \\
\hline & & Home & 98 & 19.6 \\
\hline & & Can't say & 69 & 13.8 \\
\hline \multirow{3}{*}{10} & \multirow{3}{*}{$\begin{array}{l}\text { Are you aware of any infection during } \\
\text { pregnancy that can causes have to your } \\
\text { baby }\end{array}$} & Don't know & 47 & 9.4 \\
\hline & & No & 180 & 36.0 \\
\hline & & Yes & 266 & 53.2 \\
\hline \multirow{3}{*}{11} & \multirow{3}{*}{$\begin{array}{l}\text { Are you aware of any medicines other than } \\
\text { prescribed by doctor can cause have to your } \\
\text { baby }\end{array}$} & Can't say & 38 & 7.6 \\
\hline & & No & 220 & 44.0 \\
\hline & & Yes & 224 & 44.8 \\
\hline \multirow{15}{*}{12} & \multirow{15}{*}{$\begin{array}{l}\text { What are the danger signals of pregnancy? } \\
\text { A: excessive vomiting, } \\
\text { B: persistent swelling of limbs, } \\
\text { C: vaginal bleeding/discharge, } \\
\text { D: convulsions, } \\
\text { E: week or no fetal movements, } \\
\text { F: visual disturbances, } \\
\text { G: headache, } \\
\text { H: pain abdomen. }\end{array}$} & A & 18 & 3.6 \\
\hline & & A-D, $\mathrm{H}$ & 1 & .2 \\
\hline & & $\mathrm{A}, \mathrm{B}$ & 7 & 1.4 \\
\hline & & $\mathrm{A}, \mathrm{B}, \mathrm{C}$ & 3 & 0.6 \\
\hline & & $\mathrm{A}, \mathrm{B}, \mathrm{C}, \mathrm{D}$ & 11 & 2.2 \\
\hline & & $\mathrm{A}, \mathrm{B}, \mathrm{C}, \mathrm{D}, \mathrm{E}$ & 4 & 0.8 \\
\hline & & $\mathrm{A}, \mathrm{B}, \mathrm{C}, \mathrm{D}, \mathrm{E}, \mathrm{F}$ & 1 & 0.2 \\
\hline & & $\mathrm{A}, \mathrm{B}, \mathrm{C}, \mathrm{D}, \mathrm{G}, \mathrm{H}$ & 1 & 0.2 \\
\hline & & $\mathrm{A}, \mathrm{B}, \mathrm{C}, \mathrm{D}, \mathrm{H}$ & 5 & 1.0 \\
\hline & & $\mathrm{A}, \mathrm{B}, \mathrm{C}, \mathrm{F}, \mathrm{G}, \mathrm{H}$ & 2 & 0.4 \\
\hline & & $\mathrm{A}, \mathrm{B}, \mathrm{C}, \mathrm{G}$ & 1 & 0.2 \\
\hline & & $\mathrm{A}, \mathrm{B}, \mathrm{C}, \mathrm{G}, \mathrm{H}$ & 2 & 0.4 \\
\hline & & $\mathrm{A}, \mathrm{B}, \mathrm{C}, \mathrm{H}$ & 5 & 1.0 \\
\hline & & $\mathrm{A}, \mathrm{B}, \mathrm{D}$ & 1 & 0.2 \\
\hline & & $\mathrm{A}, \mathrm{B}, \mathrm{D}, \mathrm{G}$ & 1 & 0.2 \\
\hline
\end{tabular}




\begin{tabular}{|c|c|c|}
\hline Options & Frequency & Percentage \\
\hline $\mathrm{A}, \mathrm{B}, \mathrm{D}, \mathrm{F}$ & 3 & 0.6 \\
\hline $\mathrm{A}, \mathrm{B}, \mathrm{D}, \mathrm{F}, \mathrm{H}$ & 1 & 0.2 \\
\hline $\mathrm{A}, \mathrm{B}, \mathrm{D}, \mathrm{G}$ & 3 & 0.6 \\
\hline $\mathrm{A}, \mathrm{B}, \mathrm{D}, \mathrm{H}$ & 1 & 0.2 \\
\hline $\mathrm{A}, \mathrm{B}, \mathrm{E}, \mathrm{F}, \mathrm{G}, \mathrm{H}$ & 1 & 0.2 \\
\hline $\mathrm{A}, \mathrm{B}, \mathrm{E}, \mathrm{H}$ & 1 & 0.2 \\
\hline $\mathrm{A}, \mathrm{B}, \mathrm{F}, \mathrm{G}$ & 1 & 0.2 \\
\hline $\mathrm{A}, \mathrm{B}, \mathrm{G}, \mathrm{H}$ & 1 & 0.2 \\
\hline $\mathrm{A}, \mathrm{B}, \mathrm{G}, \mathrm{H}$ & 1 & 0.2 \\
\hline $\mathrm{A}, \mathrm{B}, \mathrm{H}$ & 8 & 1.6 \\
\hline $\mathrm{A}, \mathrm{C}$ & 2 & 0.4 \\
\hline $\mathrm{A}, \mathrm{C}, \mathrm{D}$ & 4 & 0.8 \\
\hline $\mathrm{A}, \mathrm{C}, \mathrm{D}, \mathrm{F}, \mathrm{G}$ & 1 & 0.2 \\
\hline $\mathrm{A}, \mathrm{C}, \mathrm{D}, \mathrm{F}, \mathrm{H}$ & 1 & 0.2 \\
\hline $\mathrm{A}, \mathrm{C}, \mathrm{D}, \mathrm{G}$ & 2 & 0.4 \\
\hline $\mathrm{A}, \mathrm{C}, \mathrm{D}, \mathrm{H}$ & 4 & 0.8 \\
\hline $\mathrm{A}, \mathrm{C}, \mathrm{D}, \mathrm{G}$ & 1 & 0.2 \\
\hline $\mathrm{A}, \mathrm{C}, \mathrm{D}, \mathrm{A}$ & 1 & 0.2 \\
\hline $\mathrm{A}, \mathrm{C}, \mathrm{E}$ & 3 & 0.6 \\
\hline $\mathrm{A}, \mathrm{C}, \mathrm{F}, \mathrm{H}$ & 1 & 0.2 \\
\hline $\mathrm{A}, \mathrm{C}, \mathrm{G}$ & 1 & 0.2 \\
\hline $\mathrm{A}, \mathrm{C}, \mathrm{G}, \mathrm{H}$ & 1 & 0.2 \\
\hline $\mathrm{A}, \mathrm{C}, \mathrm{H}$ & 7 & 1.4 \\
\hline A, D & 1 & 0.2 \\
\hline $\mathrm{A}, \mathrm{D}, \mathrm{E}$ & 2 & 0.4 \\
\hline $\mathrm{A}, \mathrm{D}, \mathrm{F}$ & 2 & 0.4 \\
\hline $\mathrm{A}, \mathrm{D}, \mathrm{F}, \mathrm{G}$ & 2 & 0.4 \\
\hline $\mathrm{A}, \mathrm{D}, \mathrm{F}, \mathrm{H}$ & 1 & 0.2 \\
\hline $\mathrm{A}, \mathrm{D}, \mathrm{G}$ & 4 & 0.8 \\
\hline $\mathrm{A}, \mathrm{D}, \mathrm{H}$ & 3 & 0.6 \\
\hline $\mathrm{A}, \mathrm{E}, \mathrm{H}$ & 3 & 0.6 \\
\hline $\mathrm{A}, \mathrm{F}, \mathrm{G}, \mathrm{H}$ & 1 & 0.2 \\
\hline $\mathrm{A}, \mathrm{G}$ & 1 & 0.2 \\
\hline $\mathrm{A}, \mathrm{H}$ & 9 & 1.8 \\
\hline All & 32 & 6.4 \\
\hline B & 33 & 6.6 \\
\hline $\mathrm{B}, \mathrm{C}$ & 17 & 3.4 \\
\hline $\mathrm{B}, \mathrm{C}, \mathrm{D}$ & 12 & 2.4 \\
\hline $\mathrm{B}, \mathrm{C}, \mathrm{D}, \mathrm{E}, \mathrm{F}, \mathrm{H}$ & 1 & 0.2 \\
\hline $\mathrm{B}, \mathrm{C}, \mathrm{D}, \mathrm{F}$ & 1 & 0.2 \\
\hline $\mathrm{B}, \mathrm{C}, \mathrm{E}, \mathrm{H}$ & 3 & 0.6 \\
\hline $\mathrm{B}, \mathrm{C}, \mathrm{F}$ & 1 & 0.2 \\
\hline $\mathrm{B}, \mathrm{C}, \mathrm{G}$ & 2 & 0.4 \\
\hline $\mathrm{B}, \mathrm{C}, \mathrm{G}, \mathrm{H}$ & 1 & 0.2 \\
\hline $\mathrm{B}, \mathrm{C}, \mathrm{H}$ & 3 & 0.6 \\
\hline $\mathrm{B}, \mathrm{D}$ & 1 & 0.2 \\
\hline $\mathrm{B}, \mathrm{D}, \mathrm{F}, \mathrm{G}$ & 3 & 0.6 \\
\hline $\mathrm{B}, \mathrm{D}, \mathrm{G}$ & 2 & 0.4 \\
\hline $\mathrm{B}, \mathrm{D}, \mathrm{H}$ & 5 & 1.0 \\
\hline $\mathrm{B}, \mathrm{E}$ & 1 & 0.2 \\
\hline $\mathrm{B}, \mathrm{E}, \mathrm{F}$ & 1 & 0.2 \\
\hline $\mathrm{B}, \mathrm{E}, \mathrm{G}$ & 1 & 0.2 \\
\hline $\mathrm{B}, \mathrm{E}, \mathrm{H}$ & 3 & 0.6 \\
\hline $\mathrm{B}, \mathrm{F}$ & 2 & 0.4 \\
\hline
\end{tabular}




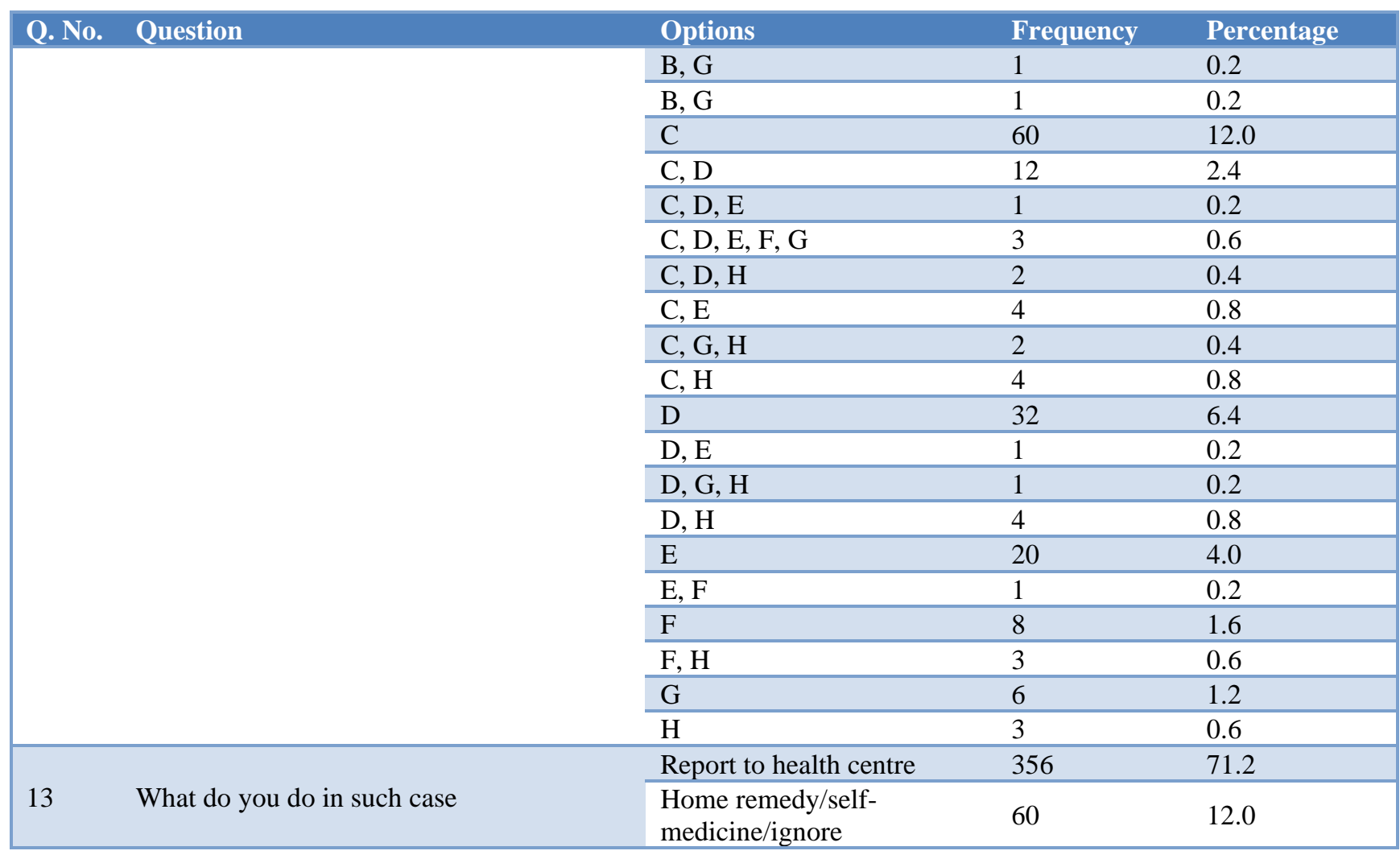

\section{Reason-home delivery is better than hospital delivery}

When they asked reason about delivery at home, 3.6\% responded transportation problem, $2.8 \%$ responded easy labour, others were responded comfortable conditions at home, feel shy, no co-operation in the family, no nearby health facilities, and not aware of medical facilities (Table 3).

When pregnant asked reason for hospital delivery, 30.2\% responded for a healthy child delivery, $9.8 \%$ responded to nearby health facility, 5.8\% responded to free care and 4.4\% responded good Hospital Services, and others were responded to On health workers motivation, nearby facility, and encouraging family (Table 3).

Table 4 shows the knowledge of the respondents on antenatal care. $71.6 \%$ of them responded that pregnant women need to go for ante natal check-up during $1^{\text {st }}-3^{\text {rd }}$ months.

According to above graph, maximum no. of respondents (35.2\%) are those who thought that during pregnancy the TT injection given to pregnant women is very important for care of both mother and baby, $32 \%$ respondents are those who stated that the TT injection given to pregnant women is very important for care of baby, $11 \%$ respondents don't know anything about importance of TT vaccination and only $20.2 \%$ respondents stated that the TT vaccine given to pregnant women is very important for mother (Figure 4).

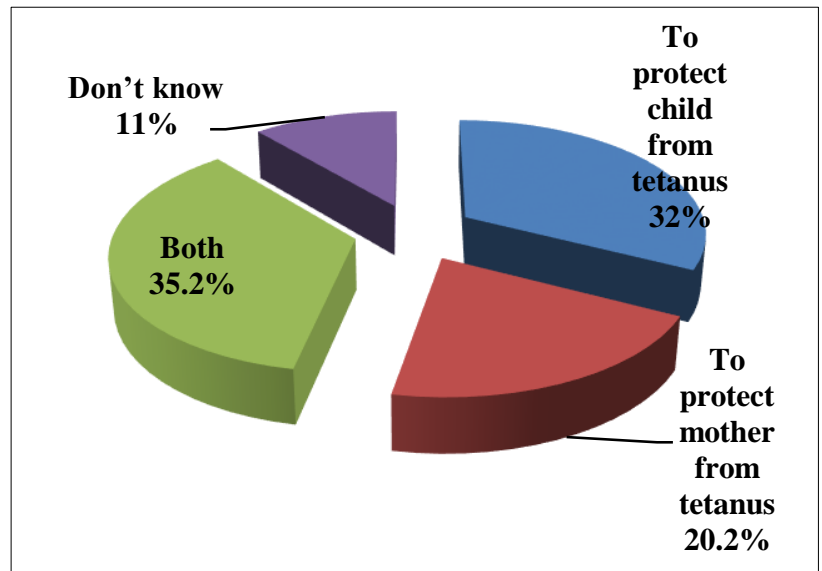

Figure 4: Importance of TT injection.

Ninety-two percent of the study population responded that Injection tetanus toxoid (TT) should be given during pregnancy but $3.2 \%$ told don't know. $65 \%$ of these population given 2 tetanus toxoid doses required to immunise against tetanus during pregnancy. That means $65 \%$ knew the correct dose.

A total $2.2 \%$ of pregnant undergone all tests related to infections, grouping etc. $53.2 \%$ pregnant known about the aware of any infection during pregnancy that can affects to the baby, $36 \%$ pregnant responded no knowledge and $9.4 \%$ responded completely don't know about it. 
Also, $70.2 \%$ of them reckoned that Iron folic acid is necessary during pregnancy and they knew correct doses needed.

A total $24.2 \%$ pregnant have the knowledge about iron and folate to increase blood of mother, $17.2 \%$ known these two will be used to relieve weakness, $46 \%$ known about the total benefits of iron and folate and remaining $10.2 \%$ doesn't know about these medications.

Danger signs like excessive vomiting, persistent swelling of limbs, vaginal bleeding/discharge, convulsions, week or no fetal movements, visual disturbances, headache, pain abdomen were observed in the studied pregnant. Upon these $12 \%$ shown vaginal bleeding/discharge, $6.4 \%$ shown convulsions, $6.4 \%$ shown all complaints, and $6.6 \%$ shown persistent swelling of limbs. In such case, $71.2 \%$ pregnant females reported that they know about the danger signs during pregnancy and these danger signs required immediate consultation with doctor or health care providers and $12 \%$ responded that these signs should be ignored (Table 4).

A total $50.4 \%$ of pregnant were advised to diet Intake of green vegetable, $21.8 \%$ advised Intake of milk, $22.8 \%$ advised intake of fruits, and 3.2\% advised Intake of meat to change to be made in diet to get complete dietary requirements.

Financial reasons, unavailability of transport facility and thinking 'it is not necessary' were the main reasons for not accessing antenatal care.

\section{DISCUSSION}

Knowledge not only transforms, but also empowers women and improves their self-esteem. It is expected that educated women are more likely to be aware about their health status and seek health knowledge. Women who married after 18 years had more knowledge than those who got married earlier. This may be explained by the fact that those who got married earlier had less schooling than those who married later.

A total $86 \%$ lying between 20 to 29 years age group followed by 30 to 39 -year age group (5\%), > 40-year age group $(5 \%)$, and < 20-year age group (4\%). Another study from Bangladesh revealed maternal age as one of the important predictors for ANC. ${ }^{6}$ It was found that higher level of education, age at marriage and living in owned house were statistically associated with better knowledge. ${ }^{7}$

A total $71.4 \%$ possess their multiverdia and $27.6 \%$ pregnant women are those who possess first pregnancy.

Chandhiok $\mathrm{N}$ et al conducted a study on antenatal care utilization in different part of India found that $73.9 \%$ of women had completed 3 antenatal visits. ${ }^{8}$
In this study, 20.6\% women responded that they had completed 3 antenatal visits, $26.6 \%$ are those who thought that at least more than 5 ANC checkup should be done during pregnancy, and $9.8 \%$ respondents are who thought that at least 5 ANC checkup should be done during pregnancy. In another study at least one antenatal clinic visit were made by $97.4 \%$ of pregnant women. ${ }^{9} \mathrm{~A}$ study done by Khatib $\mathrm{N}$ et al, in which the minimum 3 antenatal visits made by pregnant female is $33.6 \% .^{10}$

The knowledge of antenatal care and its importance was known to $82.8 \%$ women. ${ }^{11}$ In our study $96.1 \%$ pregnant females responded that registration for antennal care and visits is essential, in those $34.5 \%$ were Strongly agreed, $46.2 \%$ were agreed, and $15.2 \%$ were neutral.

Shafqat $\mathrm{T}$ et al, reported that $83.98 \%$ women had awareness regarding appropriate diet and $80.85 \%$ had knowledge regarding iron and vitamin supplementation during pregnancy. ${ }^{11}$ According to a study done by Mithra et al in urban area of South India around $73.2 \%$ were aware of the importance of IFA tablets during pregnancy and only $58.7 \%$ consumed all the IFA tablets given to them. ${ }^{12}$

In this study, maximum no. of respondents (87.2\%) are take iron and folic acid tablets during pregnancy and $11 \%$ respondents do not take iron and folic acid tablets during pregnancy. $24.2 \%$ pregnant have the knowledge about iron and folate to increase blood of mother, $17.2 \%$ known these two will be used to relieve weakness, $46 \%$ known about the total benefits of iron and folate and remaining $10.2 \%$ don't know about these medications. A similar type of study done by Pal PP et al in a rural area of India, showed that around $60 \%$ consumed the IFA tablets regularly and adequately. ${ }^{13}$ Due to the increased demand, consumption of IFA tablets during pregnancy reduces the Iron deficiency anaemia among pregnant women and thereby reducing anaemia related death during pregnancy.

A study done by Chandhiok $\mathrm{N}$ et al in rural areas of India, showed that among those who had antenatal care, 91.3\% had awareness about the importance of TT immunization during pregnancy. ${ }^{8}$

In this study, 92\% pregnant responded that Injection tetanus toxoid (TT) should be given during pregnancy but $3.2 \%$ told don't know. $65 \%$ of these population given 2 tetanus toxoid doses required to immunize against tetanus during pregnancy. That means $65 \%$ knew the correct dose.

A study done by Mathews et al, in rural Karnataka, showed around 97.5 had TT administered. ${ }^{14}$

Due to the changing hormone levels, fatigue and sleep problems are more common during pregnancy. It is thus necessary to adequate rest and sleep during pregnancy for positive outcomes. 
In our study only, when they asked about resting period, $24.2 \%$ responded 5-6 hours, $35.4 \%$ responded $7-8$ hours, $35.6 \%$ responded $>8$ hours per day to rest they are taking.

In another study, $38.96 \%$ were aware about the importance of sleep and rest during pregnancy. ${ }^{15}$

In our study $55.8 \%$ pregnant females responded that they had taken decision to give birth the baby in the hospital.

In another study, hospital as a place of delivery was favored by $82.03 \%$ women while $17.96 \%$ women preferred to deliver at homes and private clinics. ${ }^{11}$

The complications arise due to hypertension was not known by $39.8 \%$ of the women and also, they don't know that women with high blood pressure will affect the fetal growth in pregnancy. ${ }^{16}$ In our study, $71.2 \%$ pregnant females reported that they knew about the danger signs during pregnancy. These pregnant females had mentioned the danger signs during pregnancy as excessive vomiting, persistent swelling of limbs, vaginal bleeding/discharge, convulsions, week or no fetal movements, visual disturbances, headache, pain abdomen. When they asked about any ailment, $61.6 \%$ responded no and $38.4 \%$ responded yes that they had hypertension or diabetes or Thyroid problem or anemia problems.

The reasons for not attending antenatal check-up were financial reasons, illiterate (15.4\%), and unaware about ANC. This finding is consistent with the findings of Sanjel $\mathrm{S}$ et al. ${ }^{17}$ Another study from China reported the financial difficulties as the most important reason for not attending ANC. ${ }^{18}$ Poor women usually have poor access to education, including health education due to lack of financial resources, early marriage and pregnancy, household responsibilities and unwillingness to invest in the hidden costs of education like fees, transport, etc.

The practices and attitudes are better in percentage than knowledge component.

The limitations of the present study were that it was conducted in a small number of pregnant female, the participants were of hospital based study.

To conclude, the study detected $96.1 \%$ pregnant female responded to attend antenatal check-up and its importance. The study recommends strengthening of health education by health care providers to improve the knowledge during pregnancy and post-partum period, and early reporting of danger signs.

\section{CONCLUSION}

Mother and child are considered as one unit. Utilisation of proper utilisation of antenatal care reduces both maternal and neonatal mortality. Women who are attempting for second or more than second pregnancy, they have more awareness and knowledge about ANC check-ups during pregnancy. 15.4 respondents are illiterate so they are not aware about their health during their pregnancy. Regarding delivery, some of the respondents are delivered their baby at home because of own fair of miscarriage or risk at hospital.

To improve community awareness on ANC, information, education and communication activities should be increased on ANC through community campaign and mass media like local television channel, radio and local newspapers. There is a need to motivate women to utilize maternal care services which are freely available in all the government health setups.

Funding: No funding sources

Conflict of interest: None declared

Ethical approval: The study was approved by the Institutional Ethics Committee

\section{REFERENCES}

1. Carroli G, Rooney C, Villar J. How effective is antenatal care in preventing maternal mortality and serious morbidity? An overview of the evidence. Paediatr Perinatal Epidemiol. 2001;15:1-42.

2. Gupta RK, Shora TN, Verma AK, Jan R. Knowledge regarding antenatal care services, its utilization, and delivery practices in mothers (aged 15-49 years) in a rural area of North India. Trop J Med Res. 2015;18(2):89.

3. Patil MS, Vedpathak VL, Aswar NR, Deo DS, Dahire PL. Birth preparedness and complication readiness among primigravida women attending tertiary care hospital in a rural area. Int J Comm Med Pub Health. 2017;3(8):2297-304.

4. Kabakyenga JK, Östergren PO, Turyakira E, Pettersson KO. Knowledge of obstetric danger signs and birth preparedness practices among women in rural Uganda. Reproductive Health. 2011;8(1):33.

5. Manna PK, De D GD. Knowledge, attitude and practices for antenatal care and delivery of the mothers of tea garden in Jalpaiguri and Darjeeling districts, West Bengal. National J Comm Med. 2011;2(1):4-8.

6. Koenig MA, Fauveau V, Chowdhury AI, Chakraborty J, Khan MA. Maternal mortality in Matlab, Bangladesh: 1976-85. Studies in Family Planning. 1988;19(2):69-80.

7. Punyatoya B. Knowledge, attitude and practices among pregnant women about antenatal care, danger sign during pregnancy and adopting family planning method. J Prevent Med Holistic Health. 2018;4(1):10-5.

8. Chandhiok N, Dhillon BS, Kambo L, Saxena NC. Determinants of antenatal care utilisation in rural areas of India. A cross - sectional study from 28 districts (An ICMR task force study). J Obstet Gynecol India. 2006;56:47-52. 
9. Laishram J, Thounaojam UD, Panmei J, Mukhia S, Devi HS. Knowledge and practice of antenatal care in an urban area. Indian Med Gazette. 2013;101-6.

10. Khatib N, Zhiruddin QS, Gaidhane AM. Predictors for antenatal services and pregnancy outcome in a rural area: a prospective study in Wardha district, India. Indian J Med Sci. 2009;63:436-44.

11. Shafqat T, Fayaz S, Rahim R, Saima S. Knowledge and awareness regarding antenatal care and delivery among pregnant women. J Med Sci. 2015;23:88-91.

12. Mithra P, Unnikrishnan B, Rekha T, Nithin K, Mohan K, Kulkarni V, et al. Compliance with ironfolic acid (IFA) therapy among pregnant women in an urban area of south India. African Health Sci. 2013;13(4):880-5.

13. Pal PP, Sharma S, Sarkar TK, Mitra P. Iron and folic acid consumption by the ante-natal mothers in a rural area of India in 2010. Inter J Prevent Med. 2013;4(10):1213.

14. Matthews Z, Mahendra S, Kilaru A, Ganapathy S. Antenatal care, care-seeking and morbidity in rural Karnataka, India: results of a prospective study. Asia Pacif Pop J. 2001;16(2):11-28.

15. Chellaiyan VG. A study on compliance of antenatal care among the mothers attending immunisation clinic of Murshidabad Medical College and Hospital, West Bengal. IOSR J Dent Med Sci. 2018;17(3):625 .

16. Lilungulu AG, Matovelo D, Gesase A. Reported knowledge, attitude and practice of antenatal care services among women in Dodoma Municipal, Tanzania. J Pediatr Neonatal Care. 2016;4:00125.

17. Sanjel S, Ghimire RH, Pun K. Antenatal care practices in Tamang community of hilly area in central Nepal. Kathmandu Univ Med J. 2011;34(2):57-61.

18. Zhao Q, Kulane A, Gao Y, Xu B. Knowledge and attitude on maternal health care among rural-to-urban migrant women in Shanghai, China. BMC Women's Health. 2009;9(1):5.

Cite this article as: Sitalakshmi V, Bavyasri $\mathrm{P}$, Talapala R, Kopperla M. Study on knowledge, attitude and practice of ante-natal care among pregnant women attending antenatal tertiary care institution. Int J Reprod Contracept Obstet Gynecol 2020;9:1169-80. 